\title{
Efficient Sensorless Speed Estimation of Electrical Servo Drives Using a Full-Order Nonsingular Terminal Sliding Mode Observer
}

\author{
Tan-No Nguyen, ${ }^{1,2}$ Thanh-Binh Pham, ${ }^{1,2}$ Van-Trong Hoang, ${ }^{1,2}$ Tan-Tien Nguyen, ${ }^{3}$ \\ Viet-Long Nguyen, ${ }^{1,4}$ and Nguyen-Vu Truong $\mathbb{D}^{1}$ \\ ${ }^{1}$ Institute of Applied Mechanics and Informatics, Vietnam Academy of Science and Technology, Hanoi, Vietnam \\ ${ }^{2}$ Graduate University of Science and Technology, Vietnam Academy of Science and Technology, Hanoi, Vietnam \\ ${ }^{3}$ HCMC University of Technology, Vietnam National University HCMC, Hochiminh City, Vietnam \\ ${ }^{4}$ Becamex IDC Corp, Binh Duong, Vietnam
}

Correspondence should be addressed to Nguyen-Vu Truong; nvtruong0427@gmail.com

Received 31 July 2020; Revised 30 November 2020; Accepted 29 December 2020; Published 13 January 2021

Academic Editor: Alessandro Lo Schiavo

Copyright (c) 2021 Tan-No Nguyen et al. This is an open access article distributed under the Creative Commons Attribution License, which permits unrestricted use, distribution, and reproduction in any medium, provided the original work is properly cited.

\begin{abstract}
This paper proposes an efficient sensorless speed estimation approach for electric servo drives based on the full-order nonsingular terminal sliding mode observer (FONTSM) with the application of DC motor drives. In this method, a specific full-order terminal sliding mode manifold is utilised for the observer design which results in the elimination of the chattering and avoiding the singularity phenomenon of conventional and terminal sliding modes. Here, speed and armature back emf can be directly estimated from the relevant observer's inputs which are continuous instead of being discontinuous high-frequency "switching" signals. The efficiencies and advantages of this approach have been proven and validated in both simulation and experimental results.
\end{abstract}

\section{Introduction}

Electrical drives play vital importance in many industrial applications, household appliances, factory automation, precision machine and robotics, automotives, and so on. Typically, a precision servo drive requires an efficient control strategy and relevant sensors to measure both motor's mechanical speed and position such as encoders and revolvers as well as its electrical quantities such as current sensors and voltage sensors. Nevertheless, in the past decades, the open literature has reported a substantial amount of research in sensorless control of electrical drives without the need of these mechanical sensors (i.e., [1-9]). This requires an efficient technique to estimate these servo mechanical quantities, i.e., rotor position via direct measurement of stator's current and voltage.

Numerous methods have been proposed to deal with this estimation problem such as $[1,5,8-12]$, including the most recent sliding mode-based techniques which possess attractive advantages due to outstanding robustness to both disturbances and the system's uncertainties (i.e., $[1,8-10,12-14])$. Conventional sliding mode-based methods (which adopt linear sliding manifolds), however, suffer serious drawback due to the chattering phenomenon which makes the observed results not suitable to be used for control purposes. To overcome this, various approaches have been proposed, ranging from low-pass filter with phase compensation [3] and boundary layer approximation [4] to higher-order sliding mode method [12-15] and, particularly, terminal sliding mode methods [10-12, 16-25].

Conventional sliding mode control with low-pass filter or with boundary layer approximation would significantly reduce chattering but suffers loss of system's robustness and introduction of significant phase shift and steady-state errors of the estimation. Higher-order sliding mode (HOSM) methods work with the discontinuous control in its higherorder derivatives [13-15]. Here, the switching function (discontinuous control) is at the higher-order derivatives to 
avoid the chattering phenomenon. Terminal sliding mode (TSM) methods utilize nonlinear sliding manifolds, which are functions of the states and their derivatives, to ensure the system's states to converge not only in finite time but also at faster response and higher precision [10-12, 16-18]. Traditional TSM methods, however, have singularity. In order to avoid this, nonsingular terminal sliding mode (NTSM) methods have been proposed [19] and developed rapidly in the past decade [19-24].

The literature reports various works for speed estimation of electrical servo drives $[1,2,5,8-10,12,19,20]$, particularly the use of NTSM to estimate the rotor's flux and speed of PMSM motors [20]. Recently, higher-order terminal sliding mode (HOTSM) method has been reported [12] for the estimation of these quantities for induction motors (IM). The main idea of HOTSM is to incorporate integral errors of states into the terminal sliding manifolds. Nevertheless, like NTSM and TSM methods, the discontinuous switching function is embedded in first-order derivatives. As a result, the control input, in this manner, takes the second-order sliding mode control law.

In the context of servo drive control, high-frequency switching signals (chattering phenomenon) might cause not only severe mechanical damages to the motor's gear box and brushes but also deterioration to the control performance. In order to effectively avoid such chattering, this paper proposes a full-order nonsingular terminal sliding mode (FONTSM) approach to this estimation problem. By incorporating both derivative and integral errors of states into the nonsingular terminal sliding manifolds, a composite sliding manifold is formed. FONTSM deals with higherorder derivatives in comparison to such HOTSM methods. The observer inputs, therefore, would take the third-order sliding mode control law, obtained by multiple integral. Like so, the observer inputs would be continuous, and the sliding manifold would be obtained in finite time; system's states would asymptotically converge chattering-free to zero at much faster response time and higher tracking precision in comparison to both NTSM and HOTSM methods. This is the fundamental contribution of this paper.

The remainder of this paper is organized as follows. Section 2 briefly describes the model of the kind of DC motor under study. FONTSM observer-based scheme for speed estimation of the DC motor is described in Section 3. The simulation results are presented in Section 4. Section 5 reports the experimental results, demonstrating the efficiency and merits of the proposed approach. Finally, Section 6 concludes this paper.

\section{Model of DC Motors}

Consider a series-wound DC motor of which equivalent circuit is given as in Figure 1.

Here, the electrical characteristics of this DC motor are described as in the following equation:

$$
v=R i+L \frac{d i}{d t}+E,
$$

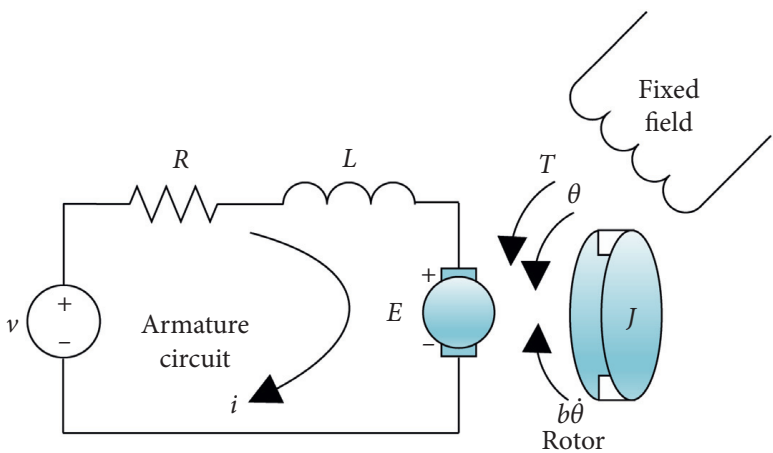

Figure 1: Equivalent circuit of the series-wound DC motor.

in which $v, i, R$, and $L$ refer to the armature voltage, current, resistance, and inductance, respectively; $E$ denotes the motor back-emf voltage which is related to the motor's shaft angular velocity $\omega$ as

$$
E=k_{e} \omega
$$

where $k_{e}$ is the back-emf voltage constant, and then

$$
\frac{\mathrm{d} i}{\mathrm{~d} t}=\frac{(-R i+v-E)}{L} .
$$

\section{Full-Order Nonsingular Terminal Sliding Mode Observer Scheme}

In this section, a full-order nonsingular terminal sliding mode observer scheme is proposed to estimate the motor shaft angular velocity of a DC motor based on the respective armature voltage and current measurement.

The current observer can be designed as follows:

$$
\frac{\mathrm{d} \hat{i}}{\mathrm{~d} t}=\frac{(-\widehat{R} \hat{i}+v+u)}{\widehat{L}} .
$$

Assuming that $\widehat{R}=R$ and $\widehat{L}=L$, the error equation, by deducting (4) from (3), takes the following form:

$$
\frac{\mathrm{d} \bar{i}}{\mathrm{~d} t}=\frac{1}{L}(-R \bar{i}+E+u) .
$$

Here, $\bar{i}=\bar{i}-i$ refers to the error of the estimation.

The full-order nonsingular terminal sliding mode (FONTSM) manifold takes the following form:

$$
l=s+\gamma \dot{s}^{p / q},
$$

and

$$
s=\bar{i}+k_{1} \int_{0}^{t} \bar{i} \mathrm{~d} \tau+k_{2} \frac{\mathrm{d} \bar{i}}{\mathrm{~d} t},
$$

which is a composite manifold by incorporating $s$ into the nonsingular terminal sliding mode (NTSM) manifold $l$. Here, $\gamma>0, p, q$ are positive odd integers, $1<p / q<2$, and $k_{1}, k_{2}$ are positive gains. The FONTSM input of the observer is designed based on the following theorem. 
Theorem 1. The current error of system (5) asymptotically converges to zero if the FONTSM manifold is chosen as (6) and (7), and the observer input is designed as follows:

$$
\begin{aligned}
& \mu_{1} \dot{u}+\mu_{2} \ddot{u}=\phi \\
& \phi=\phi_{e q}+\phi_{n} \\
& \phi_{e q}=-\lambda_{2} \overline{\bar{i}}-p / q \gamma^{-1} \dot{s}^{2-p / q} \\
& \phi_{n}=-\left(\alpha_{1}+\alpha_{2}+\beta\right) \operatorname{sign}(l)-\mu l
\end{aligned}
$$

where $\alpha_{1}, \alpha_{2}$, and $\beta$ refer to the positive control gains and $\mu$ is a positive constant with

$$
\begin{aligned}
& \lambda_{1}=k_{1}-\frac{R}{L}, \\
& \lambda_{2}=\lambda_{1}+k_{2}\left(\frac{R}{L}\right)^{2}, \\
& \mu_{1}=\frac{1}{L}\left(1-k_{2} \frac{R}{L}\right), \\
& \mu_{2}=\frac{k_{2}}{L},
\end{aligned}
$$

and the following conditions hold:

$$
\left\|\mu_{1} \dot{E}\right\|<\alpha_{1},\left\|\mu_{2} \ddot{E}\right\|<\alpha_{2}
$$

Proof. Consider a Lyapunov function candidate:

$$
V=\frac{1}{2} l^{2}
$$

Differentiating $V$ with respect to time yields

$$
\begin{aligned}
\dot{V} & =l . \dot{l}=l\left(\dot{s}+\frac{p}{q} \gamma \dot{s}^{p / q-1} \ddot{s}\right) \\
& =\frac{p}{q} l \gamma \dot{s}^{p / q-1}\left(\ddot{s}+\frac{q}{p} \gamma^{-1} \dot{s}^{2-p / q}\right) .
\end{aligned}
$$

According to (7),

$$
\begin{aligned}
\dot{s} & =\dot{\bar{i}}+k_{1} \bar{i}+k_{2} \ddot{\bar{i}} \\
& =k_{1} \bar{i}+\frac{1}{L}(-R \bar{i}+e+u)+\frac{k_{2}}{L}(-R \dot{\bar{i}}+\dot{e}+\dot{u}) \\
& =k_{1} \bar{i}+\frac{1}{L}(-R \bar{i}+e+u)+\frac{k_{2}}{L}\left(\begin{array}{c}
-R\left(\frac{-R}{L} \bar{i}+\frac{e}{L}+\frac{u}{L}\right) \\
+\dot{e}+\dot{u}
\end{array}\right) .
\end{aligned}
$$

By rearranging the above equation, it yields

$$
\begin{aligned}
\dot{s} & =\lambda_{2} \bar{i}+\mu_{1} E+\mu_{2} \dot{E}+\mu_{1} u+\mu_{2} \dot{u} \\
\ddot{s} & =\lambda_{2} \dot{\bar{i}}+\mu_{1} \dot{E}+\mu_{2} \ddot{E}+\mu_{1} \dot{u}+\mu_{2} \ddot{u} \\
& =\lambda_{2} \dot{\bar{i}}+\mu_{1} \dot{E}+\mu_{2} \ddot{E}+\phi \\
& =\lambda_{2} \dot{\bar{i}}+\mu_{1} \dot{E}+\mu_{2} \ddot{E}-\lambda_{2} \dot{\bar{i}}-\frac{p}{q} \gamma^{-1} \dot{s}^{2-p / q}+\phi_{n} \\
& =\mu_{1} \dot{E}+\mu_{2} \ddot{E}-\frac{p}{q} \gamma^{-1} \dot{s}^{2-p / q}+\phi_{n} .
\end{aligned}
$$

Therefore,

$$
\begin{aligned}
\dot{V}= & \frac{p}{q} l \gamma \dot{s}^{p / q-1}\left(\mu_{1} \dot{E}+\mu_{2} \ddot{E}+\phi_{n}\right) \\
= & \frac{p}{q} l \gamma \dot{s}^{p / q-1}\left(\mu_{1} \dot{E}+\mu_{2} \ddot{E}-\alpha_{1} \operatorname{sign}(l)-\alpha_{2} \operatorname{sign}(l)\right) \\
& -\frac{p}{q} l \gamma \dot{s}^{p / q-1}(\beta \operatorname{sign}(l)+\mu l) .
\end{aligned}
$$

In this case, $\dot{s}^{p / q-1}>0$, which means

$$
\begin{aligned}
& \frac{p}{q} l \gamma \dot{s}^{p / q-1}(\beta \operatorname{sign}(l)+\mu l) \\
& =\frac{p}{q} \gamma \dot{s}^{p / q-1}\left(\beta\|l\|+\mu\|l\|^{2}\right) \geq 0, \\
& \frac{p}{q} l \gamma \dot{s}^{p / q-1}\left(\mu_{1} \dot{E}+\mu_{2} \ddot{E}-\alpha_{1} \operatorname{sign}(l)-\alpha_{2} \operatorname{sign}(l)\right) \\
& =\frac{p}{q} \gamma \dot{s}^{p / q-1} l\left(\mu_{1} \dot{E}+\mu_{2} \ddot{E}-\alpha_{1} \operatorname{sign}(l)-\alpha_{2} \operatorname{sign}(l)\right) \\
& =\frac{p}{q} \gamma \dot{s}^{p / q-1} l\left(\mu_{1} \dot{E}-\alpha_{1} \operatorname{sign}(l)\right) \\
& \quad+\frac{p}{q} \gamma \dot{s}^{p / q-1} l\left(\mu_{2} \ddot{E}-\alpha_{2} \operatorname{sign}(l)\right) \leq 0 .
\end{aligned}
$$

As a result, $\dot{V} \leq-p / q \gamma \dot{s}^{p / q-1}\left(\beta\|l\|+\mu\|l\|^{2}\right)<0$ for all $l \neq 0$, which means the error dynamics (5) can reach $l=0$ first and then $s=0$ in finite time, and the current error $\bar{i}$ and its derivative $\bar{i}$ exponentially converge to zero.

Consequently, $e+u$ would converge to zero asymptotically, and the corresponding motor's shaft angular velocity can then be computed as

$$
\omega=\frac{1}{k_{e}} E=-\frac{1}{k_{e}} u .
$$

Remark 1. With the initial conditions $u(0)=\dot{u}(0)=0$ and $\mu_{1} \neq 0, \mu_{2} \neq 0$, the observer input would take the following mathematical form:

$$
u=\mu_{2}^{-1} e^{-\mu_{1} / \mu_{2} t} \int_{0}^{t}\left[e^{\mu_{1} / \mu_{2} t} \int_{0}^{t} \phi \mathrm{d} \tau_{1}\right] \mathrm{d} \tau_{2} .
$$


Remark 2. Suppose $t_{l}$ to be the time when $l$ reaches zero from $l\left(t_{r}\right) \neq 0$, i.e., $l(t)=0$ for all $t \geq t_{l}$.

Here,

$$
\dot{V} \leq-\frac{p}{q} \gamma \dot{s}^{p / q-1}\left(\beta\|l\|+\mu\|l\|^{2}\right) \leq-\frac{p}{q} \gamma \dot{s}^{p / q-1} \beta\|l\| .
$$

Since $\dot{s}^{p / q-1}>0, \exists \delta>0: \dot{s}^{p / q-1} \geq \delta \forall t \geq t_{r}$, meaning that

$$
\begin{aligned}
\dot{V} & \leq-\frac{p}{q} \gamma \dot{s}^{p / q-1} \beta\|l\| \leq-\frac{p}{q} \gamma \delta \beta\|l\| \\
\frac{\dot{V}}{\sqrt{2 V}} & \leq-\frac{p}{q} \gamma \delta \beta \\
\int_{t_{r}}^{t_{l}} \frac{\dot{V}}{\sqrt{2 V}} \mathrm{~d} t & \leq-\int_{t_{r}}^{t_{l}} \frac{p}{q} \gamma \delta \beta \mathrm{d} t \\
-\sqrt{2 V\left(t_{r}\right)} & \leq-\frac{p}{q} \gamma \delta \beta\left(t_{l}-t_{r}\right) \\
t_{l} & \leq t_{r}+\frac{q}{p \gamma \delta \beta}\left\|l\left(t_{r}\right)\right\|,
\end{aligned}
$$

$s$ and $\dot{s}$ will converge to zeroes in finite time, governed by the NTSM manifold. The total time to reach $s=0$ would be

$$
t_{s}=t_{l}+\frac{p}{p-q} \gamma^{q / p}\left|s\left(t_{l}\right)\right|^{p-q / p} .
$$

$\bar{i}$ and $\dot{\bar{i}}$ would asymptotically converge to zeroes, being driven by the natural response of the second-order linear dynamics described in (7). Here, we would be able to design the zero-reaching trajectories of $\bar{i}$ and $\bar{i}$ via selecting appropriate values of $k_{1}$ and $k_{2}$ gains which correspond to

Damping ratio $\xi=0.51 / \sqrt{k_{1} k_{2}}$ and natural frequency $\omega_{n}=\sqrt{k_{1}} / \sqrt{k_{2}}$ of this particular second-order linear dynamic.

For the simplicity of calculation, $\xi$ is chosen to be 1 , implying a critically damped response. In this case, for $t \geq t_{s}$, it yields

$$
\bar{i}=i\left(t_{s}\right) e^{-\omega_{n}\left(t-t_{s}\right)}+\left(\dot{\bar{i}}\left(t_{s}\right)+\omega_{n} \bar{i}\left(t_{s}\right)\right) e^{-\omega_{n}\left(t-t_{s}\right)},
$$

which asymptotically converges to zero (i.e., a very small value $\varepsilon$, close to zero) within

$$
t-t_{s}=\frac{-\ln \varepsilon}{\omega_{n}}
$$

or

$$
t=t_{l}+\frac{p}{p-q} \gamma^{q / p}\left|s\left(t_{l}\right)\right|^{p-q / p}-\frac{\ln \varepsilon}{\omega_{n}} .
$$

Remark 3. In a special case, when $k_{1}=0$ and $k_{2} \neq 0$, the error dynamics (for $t \geq t_{s}$ ) obtain the following expression:

$$
|\bar{i}(t)|=\left|\bar{i}\left(t_{s}\right)\right| e^{-\left(t-t_{s}\right) / k_{2}}
$$

and exponentially converge to zero within

$$
\begin{aligned}
t-t_{s} & =-k_{2} \ln \varepsilon \text { or } \\
t & =t_{l}+\frac{p}{p-q} \gamma^{q / p}\left|s\left(t_{l}\right)\right|^{p-q / p}-k_{2} \ln \varepsilon .
\end{aligned}
$$

Remark 4. In another special case, when $k_{1} \neq 0$ and $k_{2}=0$, the error dynamics (for $t \geq t_{s}$ ) obtain the following expression:

$$
|\bar{i}(t)|=\left|\bar{i}\left(t_{s}\right)\right| e^{-k_{1}\left(t-t_{s}\right)}
$$

and exponentially converge to zero within $t-t_{s}=-\ln \varepsilon / k_{1}$ or

$$
t=t_{l}+\frac{p}{p-q} \gamma^{q / p}\left|s\left(t_{l}\right)\right|^{p-q / p}-\frac{\ln \varepsilon}{k_{1}} .
$$

In this case, the observer input would take the following form:

$$
\begin{aligned}
u & =\mu_{1}^{-1} \int_{0}^{t}\left[\begin{array}{c}
-\lambda_{1} \dot{\bar{i}}-\frac{p}{q} \gamma^{-1} \dot{s}^{2-p / q} \\
-\left(\alpha_{1}+\beta\right) \operatorname{sign}(l)-\mu l
\end{array}\right] \mathrm{d} \tau= \\
& =-\frac{\lambda_{1}}{\mu_{1}} \bar{i}-\mu_{1}^{-1} \int_{0}^{t}\left[\begin{array}{c}
\frac{p}{q} \gamma^{-1} \dot{s}^{2-p / q} \\
+\left(\alpha_{1}+\beta\right) \operatorname{sign}(l)+\mu l
\end{array}\right] \mathrm{d} \tau .
\end{aligned}
$$

Remark 5. When $k_{1}=0$ and $k_{2}=0, s=\bar{i}$. The problem turns into a standard NTSM observer form. In this case,

$$
t=t_{l}+\frac{p}{p-q} \gamma^{q / p}\left|i\left(t_{l}\right)\right|^{p-q / p}
$$

and the corresponding observer input would be

$$
\begin{aligned}
u & =\mu_{1}^{-1} \int_{0}^{t}\left[\begin{array}{c}
-\lambda_{1} \dot{\bar{i}}-\frac{p}{q} \gamma^{-1} \dot{\bar{i}}^{2-p / q} \\
-\left(\alpha_{1}+\beta\right) \operatorname{sign}(l)-\mu l
\end{array}\right] \mathrm{d} \tau= \\
& =-\frac{\lambda_{1}}{\mu_{1}} \bar{i}-\mu_{1}^{-1} \int_{0}^{t}\left[\begin{array}{c}
\frac{p}{q} \gamma^{-1} \dot{\bar{i}}^{2-p / q} \\
+\left(\alpha_{1}+\beta\right) \operatorname{sign}(l)+\mu l
\end{array}\right] \mathrm{d} \tau .
\end{aligned}
$$

\section{Simulation Results}

Simulations were conducted in MATLAB-Simulink environment using the Runge-Kutta algorithm with a step size of $0.05 \mathrm{~ms}$. Here, the DC motor's parameters in the simulation are specified as in Table 1 .

In order to demonstrate the effectiveness and advantages of the proposed FONTSM observer, the simulation results are shown in Figure 2 in benchmark with conventional and 
TABLE 1: DC motor's parameters in the simulation.

\begin{tabular}{lcc}
\hline Armature resistance & $R$ & $1.6 \Omega$ \\
\hline Armature inductance & $L$ & $0.0052 \mathrm{H}$ \\
Back-emf voltage constant & $k_{e}$ & $0.011 \mathrm{~V} / \mathrm{rpm}$ \\
Moment of inertia & $J$ & $0.0043 \mathrm{~kg} \cdot \mathrm{m}^{2}$ \\
\hline
\end{tabular}

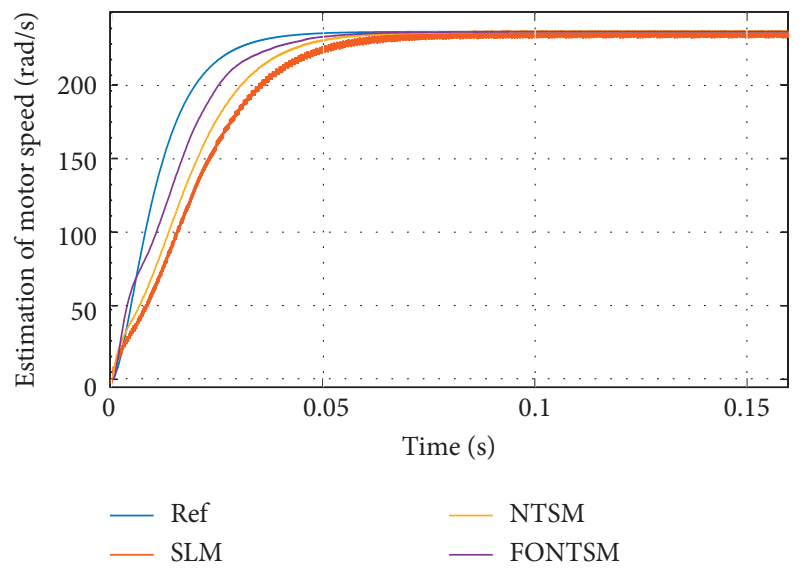

FIgUre 2: Simulation results of FONTSM (in purple) against SLM (in red) and NTSM (in yellow) observers.

nonsingular terminal sliding mode (as in Remark 5) observer methods. A zoom-in view of the simulation results at settling time is shown in Figure 3. Here, the FONTSM is designed as in Theorem 1 of which parameters are chosen as follows: $p=5, q=3, \mu=0, \quad \gamma=0.0001, \alpha_{1}+\alpha_{2}+\beta=100$, $k_{1}=250$, and $k_{2}=0.001 \quad$ (which specified $\quad \xi=1$ and $\left.\omega_{n}=500\right)$.

The merit of the proposed FONTSM observer against NTSM and conventional SLM observers is demonstrated in Figures 2-4 by a number of measures: (1) faster response time, (2) better tracking precision, and (3) chattering elimination. It can be seen that the estimated errors (Figures 5 and 6) of the FONTSM observer converge chatteringfree to zeroes within $0.047 \mathrm{~s}$, while it would be $0.06 \mathrm{~s}$ for the NTSM observer with significant chattering reduction in comparison to SLM estimated results.

\section{Experimental Results}

In order to further validate the performance of the proposed FONTSM observer design method, experiments have been carried out at our own testbed. Figures 7 and 8 show the experimental setup in which a MAE DC motor (specification as in Table 2) is controlled by a xPC target real-time workstation, a standard x86 personal computer loaded with MATLAB and Simulink xPC target real-time kernel, connected to relevant power electronics hardware, and sensors via National Instruments (PCI-6024E and PCI-6280E) DAQ cards to form a hardware-in-the-loop (HiL) simulation platform as in [26].

Here, the communication between the host PC and the target real-time workstation is via the TCP/IP protocol over RJ45 standard connection. The motor control and estimation codes are generated by MATLAB and Simulink Real-

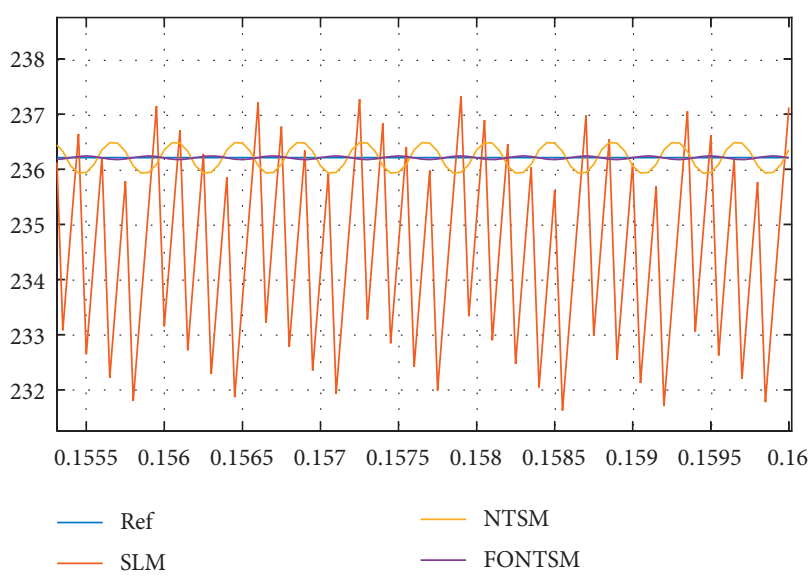

Figure 3: A zoom-in view of simulation results at settling time.

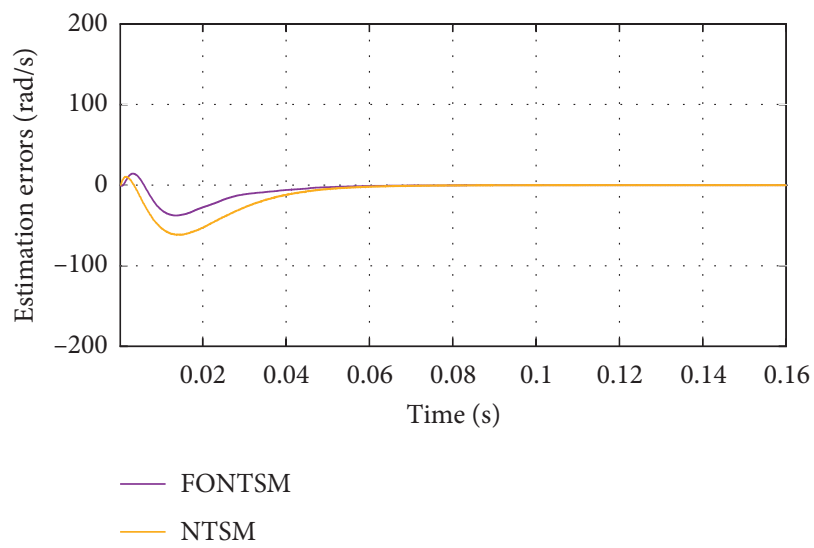

FIGURE 4: Estimation errors of the FONTSM observer versus NTSM observer.

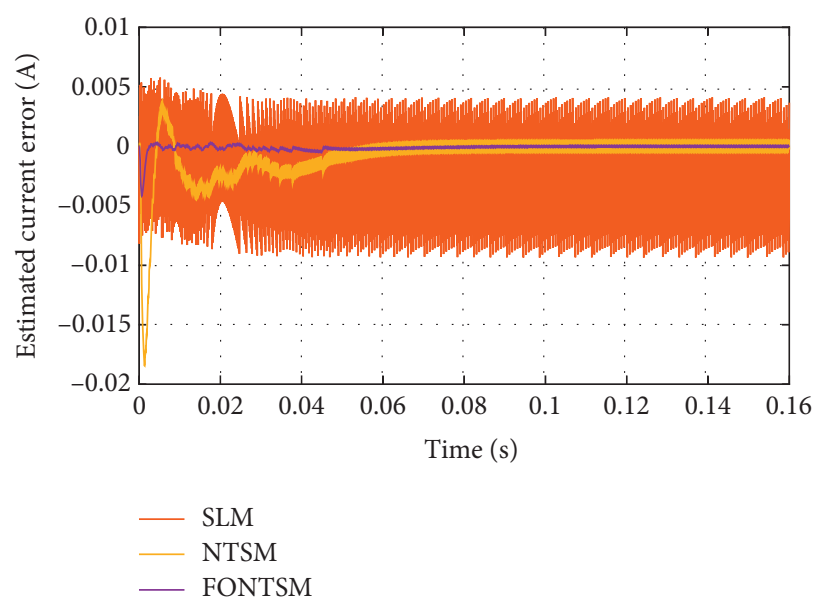

FIGURE 5: Estimation current errors of FONTSM observer against NTSM and SLM observers.

Time Workshop, compiled into machine code and loaded for real-time execution on the xPC target workstation. For the experiment, the power supply is kept at 24 volts. The sampling time of the observer is $0.05 \mathrm{~ms}$, and the PWM switching frequency of the power converter is $15 \mathrm{kHz}$. 


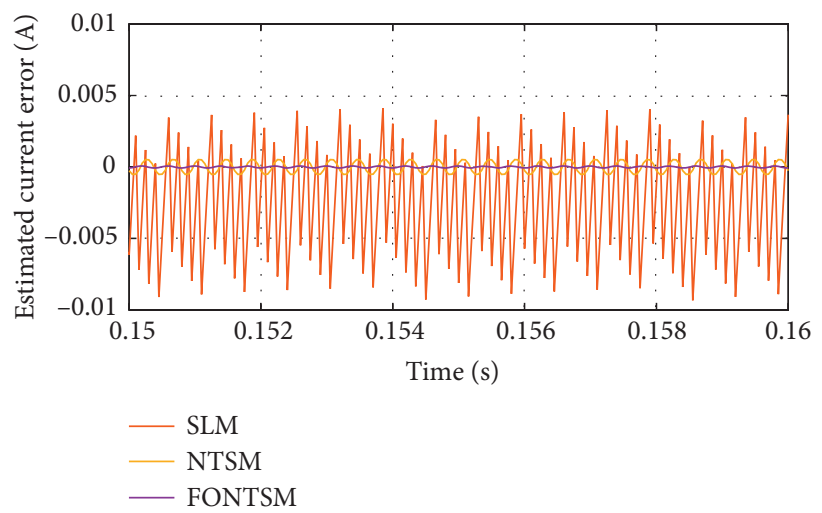

Figure 6: A zoom-in view of estimated current errors.

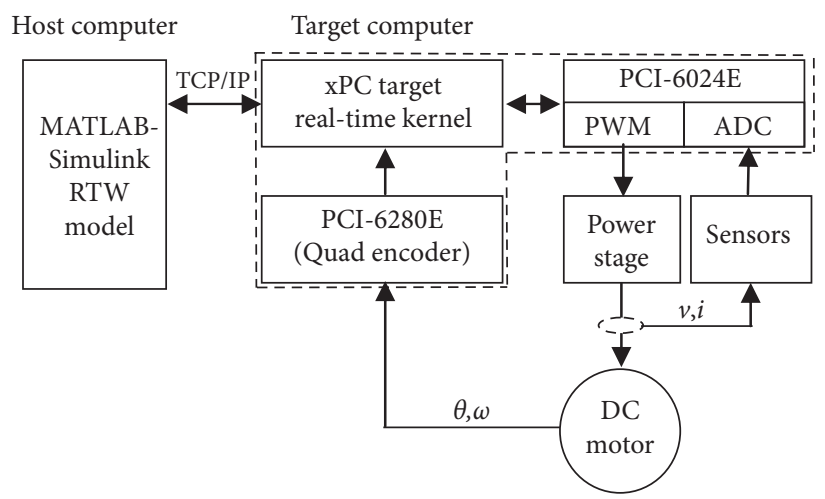

Figure 7: Experimental setup.

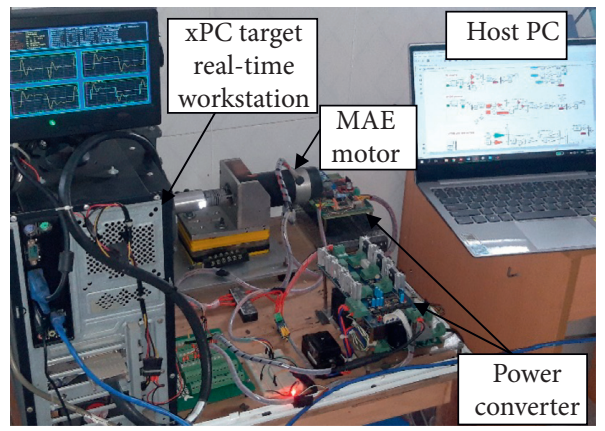

Figure 8: Experimental testbed.

TABLE 2: Motor's parameters in the experiment.

\begin{tabular}{lcc}
\hline Maximum terminal voltage & $v$ & $60 \mathrm{volts}$ \\
\hline Maximum speed & $\omega_{\max }$ & $5200 \mathrm{rpm}$ \\
Armature resistance & $R$ & $1.6 \Omega$ \\
Armature inductance & $L$ & $5.2 \mathrm{mH}$ \\
Back-emf voltage constant & $k_{e}$ & $0.011 \mathrm{~V} / \mathrm{rpm}$ \\
Moment of inertia & $J$ & $4.3 \times 10^{-4} \mathrm{~kg} \cdot \mathrm{m}^{2}$ \\
Mechanical time constant & $\tau_{M}$ & $8.2 \mathrm{~ms}$ \\
Electrical time constant & $\tau_{e}$ & $2.6 \mathrm{~ms}$ \\
\hline
\end{tabular}

In this experiment, the FONTSM observer was designed with parameters chosen to be $p=5, q=3, \mu=0$, $\gamma=0.0001, \alpha_{1}+\alpha_{2}+\beta=26, k_{1}=78.12$, and $k_{2}=0.0032$ (which specified $\xi=1$ and $\omega_{n}=156.25$ ).

The estimated speed obtained from FONTSM observer design as in Theorem 1 is overlaid against the actual speed in Figure 9. The estimated current error is shown in Figure 10, showing the estimated results converge chattering-free to zeroes within $0.04 \mathrm{~s}$. 


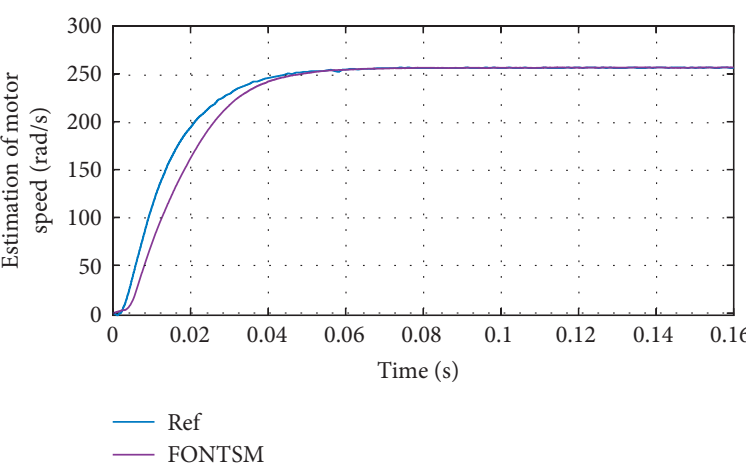

(a)

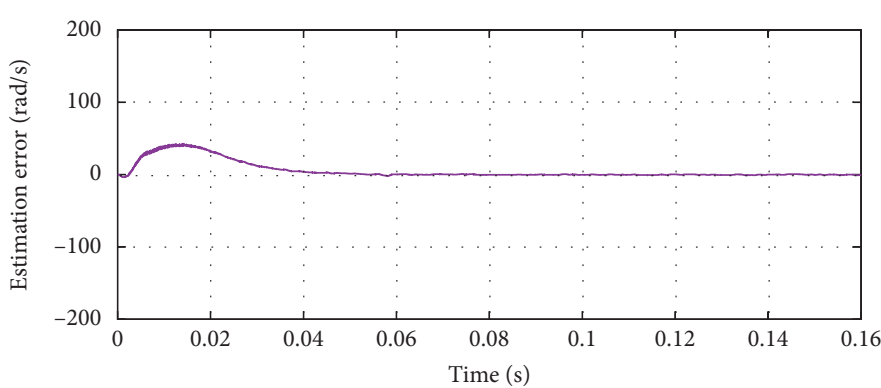

(b)

FIgURE 9: Experimental results of the FONTSM observer.

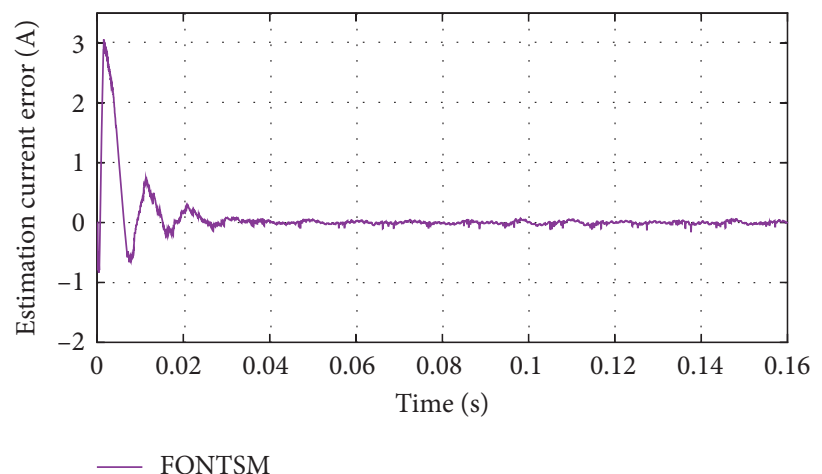

FIgURE 10: Estimated current error.

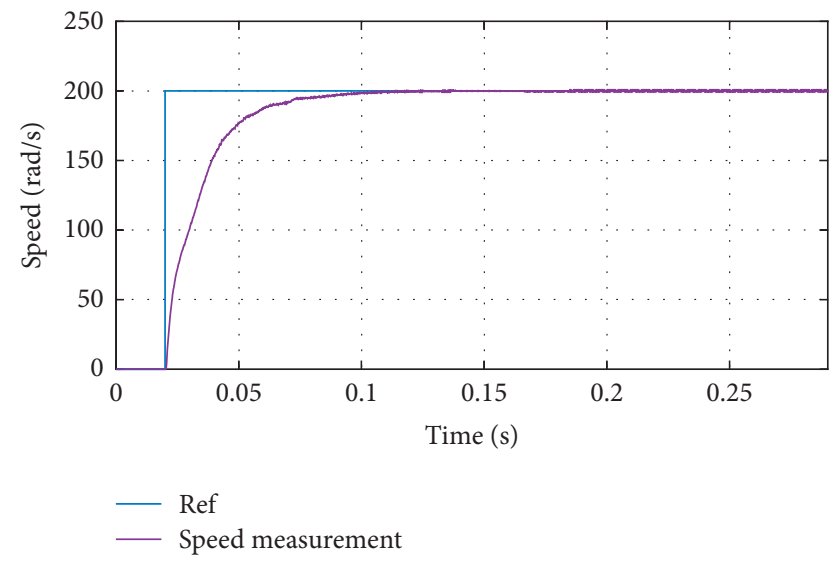

FIGURE 11: Sensorless velocity control experimental results.

Here, in order to further validate the superior merit of the proposed approach, the estimated speed, instead of the actual measurement, was fed back directly into the closed-loop control system for sensorless velocity control of the DC motor under study. Promising results are shown in Figure 11, which overlays the actual motor's angular shaft speed against the reference speed, demonstrating the effectiveness of the proposed approach.

\section{Conclusions}

This paper has proposed a full-order nonsingular terminal sliding mode observer method to estimate the motor's mechanical speed for sensorless DC motor servo drives. It relies on a composite terminal sliding manifold by incorporating both derivative and integral errors of states into the nonsingular terminal sliding manifold. In this manner, the designed FONTSM observer's input is smooth, and the estimated states converge chatteringfree to zeros both in faster response time and at better tracking precision, enabling the estimated states to be used directly as feedback in the closed-loop control system. The merit of the proposed approach has been demonstrated in simulation and experimental results in benchmark with regular NTSM and SLM observer-based methods.

Future work includes the extension of the proposed FONTSM method for sensorless speed and flux estimation of PMSM and induction motors.

\section{Data Availability}

Simulation and experimental data used in this article are available from the corresponding author upon request. 


\section{Conflicts of Interest}

The authors declare that there are no conflicts of interest regarding the publication of this paper.

\section{Acknowledgments}

This work was supported by the Vietnam Academy of Science and Technology (project no. VAST01.09/18-19).

\section{References}

[1] G. Capolino and B. Kawkabani, "Sliding mode control for electrical drives," in Proceedings of the International Electrical Machines \& Drives Conference, Hartford, CT, USA, May 2015.

[2] H. Kubota and K. Matsuse, "Speed sensorless field-oriented control of induction motor with rotor resistance adaptation," IEEE Transactions on Industry Applications, vol. 30, no. 5, pp. 1219-1224, 1994.

[3] M. Tseng and M. Chen, "Chattering reduction of sliding mode control by low pass filtering the control signal," Asian Journal of Control, vol. 12, no. 3, pp. 392-398, 2003.

[4] P. V. Suryawanshi, P. D. Shendge, and S. B. Phadke, "A boundary layer sliding mode control design for chatter reduction using uncertainty and disturbance estimator," International Journal of Dynamics and Control, vol. 4, no. 4, pp. 456-465, 2016.

[5] Y.-C. Luo and C.-L. Tsai, "Speed estimation vector-controlled induction motor drive based on fuzzy logic control flux estimator," Journal of Low Frequency Noise, Vibration and Active Control, vol. 38, no. 3-4, pp. 1220-1233, 2019.

[6] C. Lascu, I. Boldea, and F. Blaabjerg, "Direct torque control of sensorless induction motor drives: a sliding-mode approach," IEEE Transactions on Industry Applications, vol. 40, no. 2, pp. 582-590, 2004.

[7] Y. Feng, X. Yu, and F. Han, "High order terminal sliding mode observer for parameter estimation of a permanent magnet synchronous motor," IEEE Transactions on Industrial Electronics, vol. 60, no. 10, pp. 4272-4280, 2013.

[8] H. Wang, X. Ge, and Y.-C. Liu, "Second-order sliding-mode MRAS observer-based sensorless vector control of linear induction motor drives for medium-low speed maglev applications," IEEE Transactions on Industrial Electronics, vol. 65, no. 12, pp. 9938-9952, 2018.

[9] H. Kim, J. Son, and J. Lee, "A high-speed sliding-mode observer for the sensorless speed control of a PMSM," IEEE Transactions on Industrial Electronics, vol. 58, no. 9, pp. 4069-4077, 2011.

[10] M. Zhou, F. Yong, F. Han, and X. Yu, "Full-order terminal sliding-mode observer for induction motor," in Proceedings of the 2017 Chinese Control and Decision Conference, pp. 742745, Beijing, China, December 2017.

[11] Y. Feng, M. Zhou, X. Zheng, F. Han, and X. Yu, "Full-order terminal sliding-mode control of MIMO systems with unmatched uncertainties," Journal of the Franklin Institute, vol. 355, no. 2, pp. 653-674, 2018.

[12] H.-Y. Shi and Y. Feng, "High-order terminal sliding mode flux observer for induction motors," Acta Automatica Sinica, vol. 38, no. 2, pp. 288-294, 2012.

[13] S. Ding and S. Li, "Second-order sliding mode controller design subject to mismatched term," Automatica, vol. 77, pp. 388-392, 2017.

[14] A. Ferreira de Loza, L. Fridman, L. T. Aguilar, and R. Iriarte, "High-order sliding-mode observer-based input-output linearization," International Journal of Robust and Nonlinear Control, vol. 29, no. 10, pp. 3183-3199, 2019.

[15] Y. Feng, F. Han, and X. Yu, "Chattering free full-order slidingmode control," Automatica, vol. 50, no. 4, pp. 1310-1314, 2014.

[16] Y. Feng, M. Zhou, X. Zheng, F. Han, and X. Yu, "Terminal sliding-mode control of induction motor speed servo systems," in Proceedings of the IEEE International Workshop on Variable Structure Systems, pp. 351-355, Nanjing, China, July 2016.

[17] H. Du, X. Chen, G. Wen, X. Yu, and J. Lu, "Discrete-time fast terminal sliding mode control for permanent magnet linear motor," IEEE Transactions on Industrial Electronics, vol. 65, no. 12, pp. 9916-9927, 2018.

[18] Z. Ma and G. Sun, "Dual terminal sliding mode control design for rigid robotic manipulator," Journal of the Franklin Institute, vol. 355, no. 18, pp. 9127-9149, 2018.

[19] M. Zhou, Y. Feng, and X. Yu, "High-order terminal sliding observer for speed estimation of induction motors," in Proceedings of the 8th IEEE Conference on Industrial Electronics and Applications, Melbourne, Australia, April 2013.

[20] Y. Feng, J. Zheng, X. Yu, and N. V. Truong, "Hybrid Terminal Sliding Mode observer design method for a permanent magnet synchronous motor control system," IEEE Transactions on Industrial Electronics, vol. 56, no. 9, pp. 3424-3431, 2009.

[21] M. Tran and H. Kang, "Nonsingular terminal sliding mode control of uncertain second-order nonlinear systems," Mathematical Problems in Engineering, vol. 2015, Article ID 181737, 8 pages, 2015.

[22] Y. Feng, X. Yu, and F. Han, "On nonsingular terminal slidingmode control of nonlinear systems," Automatica, vol. 49, no. 6, pp. 1715-1722, 2013.

[23] S. Ding, L. Liu, and J. Park, "A novel adaptive nonsingular terminal sliding mode controller design and its application to active front steering system," International Journal of Robust and Nonlinear Control, vol. 29, no. 12, pp. 4250-4269, 2019.

[24] S. Mobayen, M. Yazdanpanah, and V. Majd, "A finite-time tracker for nonholonomic systems using recursive singularityfree FTSM," in Proceedings of the 2011 American Control Conference, San Francisco, CL, USA, February 2011.

[25] S. Mobayen and F. Tchier, "Nonsingular fast terminal sliding mode stabilizer for a class of uncertain nonlinear systems based on disturbance observer," Scientia Iranica D, vol. 24, no. 3, pp. 1410-1418, 2019.

[26] N. V. Truong, "Hardware-in-the-loop approach to controller design and testing of motion control systems using xPC target," in Proceedings of the International Conference on Intelligent and Advanced Systems, pp. 117-121, Cario, Egypt, October 2012. 